\title{
The attitude control method and realization of micro rotor-craft based on natural interaction
}

\author{
Xu Yixiong ${ }^{1, a}$, Chen Liang ${ }^{1, b}$ \\ ${ }^{1}$ South China University of Technology, School Of Design, 510006 Guangzhou, China

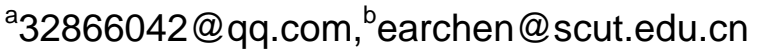

\begin{abstract}
Keywords:Natural interaction; Hand gesture controlling; Micro rotor-craft; Coordinate system
\end{abstract} transformation

\begin{abstract}
We construct a micro rotor-craft controlling method based on hand gestures of natural interaction behavior. In order to control the micro rotor-craft flight attitude by hand gestures, we obtain the hand palm flat attitude data by Leap Motion controller,and transform the data into rotor-craft attitude controlling orders between different coordinate system by coordinate system transformation and attitude angle transformation, and communicate with the micro rotor-craft by wireless transmission module.We build up a micro rotor-craft controlling system, and achieve controlling the flight actions including rising,hovering, landing, pitch and etc. by different hand gestures in real experiments.Controlling micro rotor-craft by hand gestures matches the features of natural interaction more,and it is an extension of man-machine interaction.
\end{abstract}

\section{Introduction}

Gesture recognition control is a kind of Natural User Interfaces(NUIs)[1] way. Compared with the other somatosensory interaction, gesture recognition has advantages in the natural interactive experience, rich information, high efficiency and low energy consumption. With the development of computer technology and intelligent sensor technology, the accuracy of gesture recognition has been gradually improved, and the price of hardware equipment has gradually decreased, the gesture recognition has been an important development direction of human-computer interaction.

The gesture recognition hardware can be divided into two types: Wearable recognition device and optical recognition device. With the gradually developing of technology,optical recognition device identification accuracy is relatively high with low price and friendly interaction experience. Leap motion controller(hereinafter referred as Leap Motion) is one kind of excellent optical recognition equipment[2]. Joanna C. Coelho et.al. built a virtual environment, and compare Leap Motion with traditional mouse in clicking mission on single point[3]. Lamtharn Hantrakul has used Leap Motion in audio synthesis software and interactive manipulation of real-time performance[4]. D. Bassily et al. use Leap Motion to carry out the matching test of the self-adaptable mechanical arm in order to design the remote machine control system and assisted living system for the old[5]. Boyali Ali et al. use Leap Motion to control the wheelchair to move in a complex environment[6]. In this study, a method of using gesture recognition to control a micro rotor aircraft[7] is came out and with the combination of natural interaction, the micro rotor aircraft flight attitude control technology is certificated by real experiments.

\section{System Design}

The general goal of the system is to use the hand palm plane to simulate the body plane of the aircraft in-flight. By changing the palm attitude, the aircraft flight motion(e.g. rising, landing, pitch etc.) changes accordingly. The whole system includes following four modules: gesture capture module, data processing module, wireless communication module, and micro rotor aircraft module. The overall framework of the system is shown in Fig. 1. 


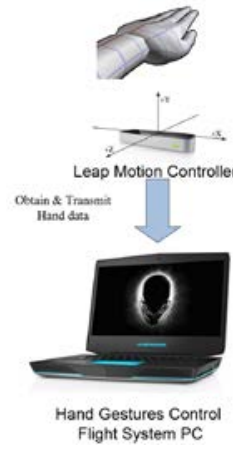

Fig. 1. System General framework

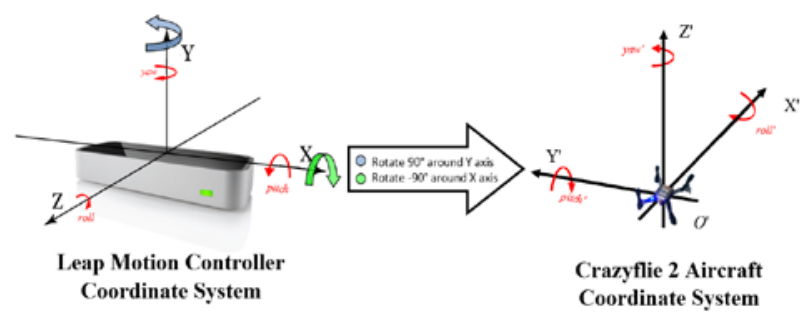

Fig. 2. Coordinate system transformation

Gesture capture module: The Leap Motion is used as a gesture data capture sensor, which is one of the desktop level optical hand gesture recognition equipment with low identification error and high frame per second(FPS)[8]. In this research, the Leap Motion is used to record the position and Euler angle of palm in real-time and transmitted it to the data processing module;Data processing module:it is a PC which communicates with Leap Motion through USB interface. This module is to deal with the original data by corresponding transformation,generate flight orders, and send to the communication module; Communication module:it is used for sending orders and receiving real-time on-board sensor data of aircraft by wireless transmission. In this research, a Crazyradio PA is used. The device uses a ISM $2.4 \mathrm{GHz}$ band radio signal, sending and receiving data packets; Micro rotor aircraft module: This is the final module of gesture control system. In this research, a Crazyflie 2 micro rotor aircraft(hereinafter referred as Crazyflie 2) is used, which is open source, easy maintenance and small size $(99 \mathrm{~mm} * 99 \mathrm{~mm}$ with the weight of $28.5 \mathrm{~g})$. The on-board single chip microcomputer sends the on-board sensors flight data to the communication module and processing errors between orders and sensors data.

\section{Key problems and realization}

Coordinate system transformation The Leap Motion coordinate system is different to the Crazyflie 2 flight coordinate system, and the definitions of the Euler angles are different either.In order to realize the system gesture interaction, it needs to establish the transformation between the two different coordinate systems. As shown in Fig. 2, according to right hand rule,the Leap Motion coordinate system firstly rotated 90 degree around the $\mathrm{Y}$ axis, and then rotated -90 degree around the $\mathrm{X}$ axis to get the Crazyflie 2 coordinate system. Coordinate system transformation matrix definition is shown as Eq. 1:

$$
\left[\begin{array}{llll}
x & y & z & 1
\end{array}\right]\left[\begin{array}{cccc}
\cos \theta & 0 & \sin \theta & 0 \\
0 & 1 & 0 & 0 \\
-\sin \theta & 0 & \cos \theta & 0 \\
0 & 0 & 0 & 1
\end{array}\right]\left[\begin{array}{cccc}
1 & 0 & 0 & 0 \\
0 & \cos (-\theta) & -\sin (-\theta) & 0 \\
0 & \sin (-\theta) & \cos (-\theta) & 0 \\
0 & 0 & 0 & 1
\end{array}\right]=\left[\begin{array}{llll}
x^{\prime} & y^{\prime} & z^{\prime} & 1
\end{array}\right] \text {. }
$$

In this study, the above equation could be simplified as Eq. 2 when $\theta=90$ degree:

$$
\left[\begin{array}{llll}
-z & -x & y & 1
\end{array}\right]=\left[\begin{array}{llll}
x^{\prime} & y^{\prime} & z^{\prime} & 1
\end{array}\right] \text {. }
$$

The Crazyflie 2 propeller rotate speed is controlled by the height of the palm $y$, which the propeller rotate speed increases when height increases,vice versa.

Attitude angle transformation it is different that the Euler angle definition of sensors and Crazyflie 2.In the Leap Motion coordinate system, the pitch angle is counter clockwise rotate around the $\mathrm{X}$ axis,the roll angle is clockwise rotate around the $\mathrm{Z}$ axis,and the yaw angle is clockwise rotate around the $\mathrm{Y}$ axis.In the Crazyflie 2 coordinate system, the pitch angle is counter clockwise rotate around the $\mathrm{Y}^{\prime}$ axis,the roll angle is counter clockwise rotate around the $\mathrm{Z}^{\prime}$ axis, and the yaw angle is counter clockwise rotate around the $\mathrm{X}^{\prime}$ axis.In order to transmit data between two coordinate systems, the transformation matrix is as Eq. 3: 


$$
\left[\begin{array}{lll}
\text { pitch roll yaw } & 1
\end{array}\right]\left[\begin{array}{cccc}
-1 & 0 & 0 & 0 \\
0 & 1 & 0 & 0 \\
0 & 0 & -1 & 0 \\
0 & 0 & 0 & 1
\end{array}\right]=\left[\begin{array}{l}
\text { pitch' roll' yaw' } \\
1
\end{array}\right] \text {. }
$$

\section{Definition of gestures and aircraft's flight attitude}

Rising and Landing: the propeller rotate speed is controlled by the palm's lifting and falling. As shown in Fig. 3, the palm lifts up, all the motors rotate speed increase simultaneously and the aircraft is rising up. Vice versa.Hovering: the palm height is fixed relatively(about $170 \mathrm{~mm}$ above the Leap Motion) and stop moving widely, all the motors rotate speed is fixed relatively and the aircraft is hovering. As shown in Fig. 4.
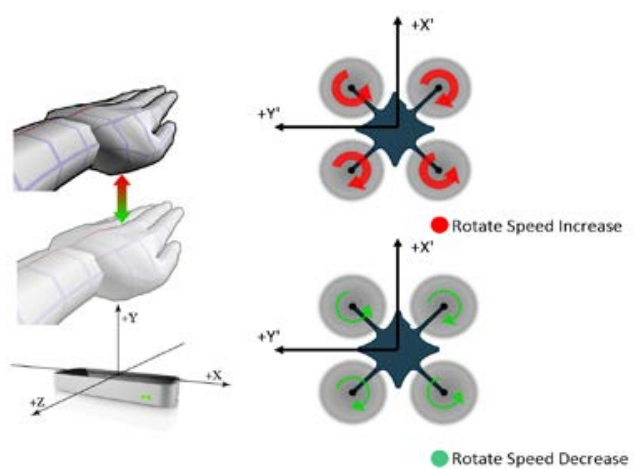

Fig. 3. Rising and landing gestures

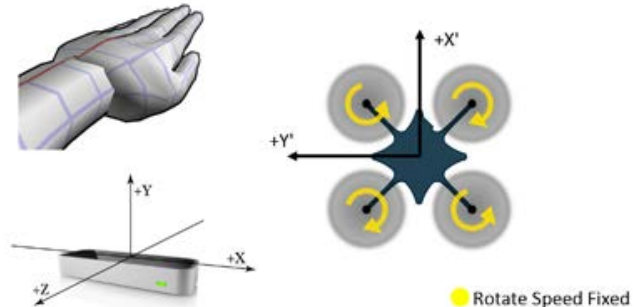

Fig. 4. Hovering gesture

Pitching:as shown in Fig. 5(a),the palm swings down, the aircraft rotates clockwise around the $\mathrm{Y}^{\prime}$ axis at an angle according to the palm swing angle.The wider the angle of palm swings down ,the wider the pitching angle of aircraft is.Vice versa.Rolling:as shown in Fig. 5(b),the palm rolls left around the $\mathrm{Z}$ axis of Leap Motion coordinate system at a certain angle, the aircraft rotates around $\mathrm{X}^{\prime}$ axis at a corresponding angle.The wider the hand palm roll angle is ,the wider the aircraft roll angle is,vice versa.Yawing:as shown in Fig. 5(c),the palm rotates around the $Y$ axis of Leap Motion at a certain angle, the aircraft yaws around $Z^{\prime}$ axis of aircraft coordinate system at a corresponding rotate speed.The wider the palm yaw angle is, the higher the aircraft yaw speed is.

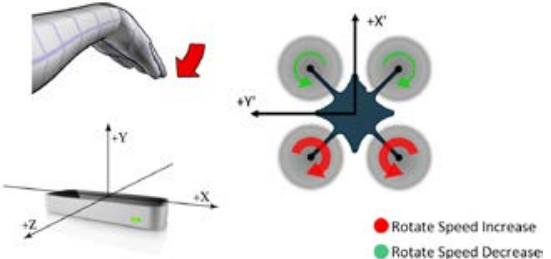

(a)pitch' down

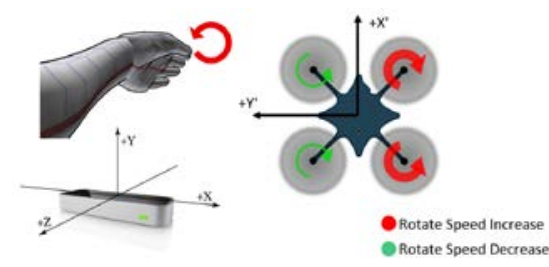

(b) roll' left

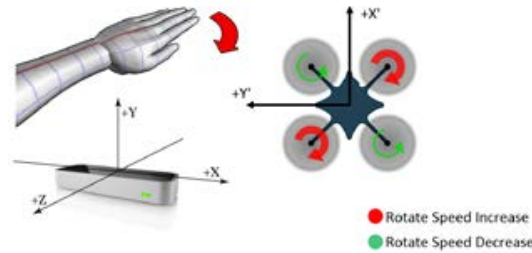

(c) yaw' right

Fig. 5. pitch' , roll' and yaw' gestures

\section{Experiments}

According to the realization methods and systematic design above, it builds up a complete flight control system and a corresponding experiment platform for the the system. The experiment results are shown in Fig. 6.The aircraft complete flight attitude:pitching from -45 degree to +45 degree, rolling from -45 degree to +45 degree and yawing from -45 degree per second to +45 degree per second.Because of the paper length limitation, the pitch +15 degree and pitch -45 degree pictures are shown in Fig. 6 (a) and Fig. 8 (b). The roll -15 degree and roll +45 degree pictures are shown in Fig. 6 (c) and Fig. 6 (d). And the yaw -15 degree/sec and yaw +45 degree/sec pictures are shown in Fig. 6 (e) and Fig. 6 (f). 


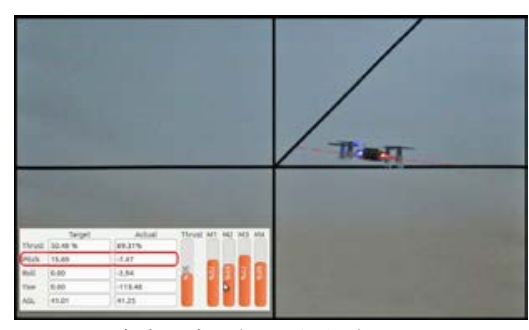

(a) pitch +15 degree

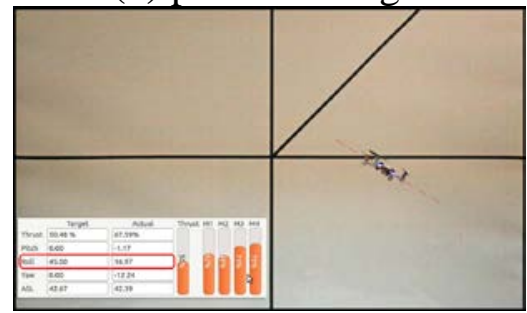

(d) roll +45 degree

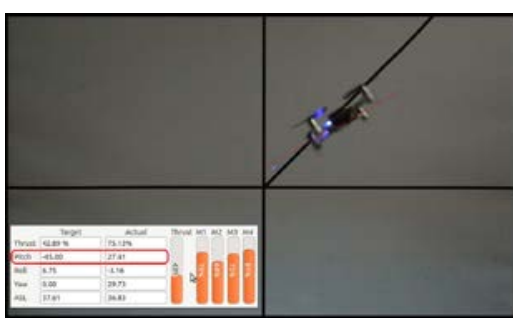

(b) pitch -45 degree

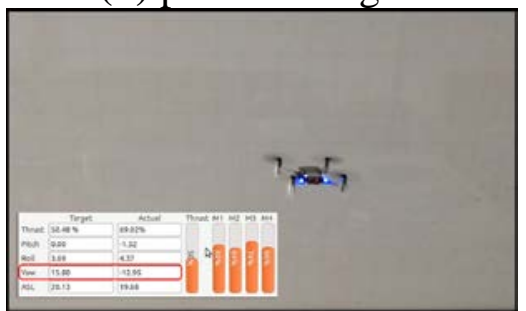

(e) yaw -15 degree/sec

Fig. 6. experiment result

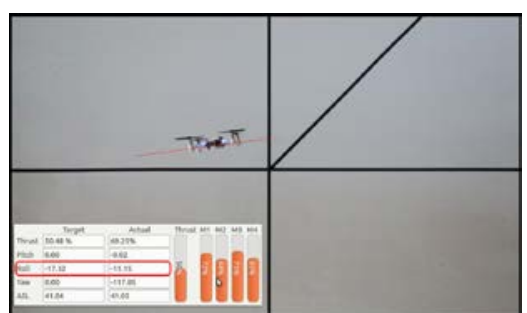

(c)roll -15 degree

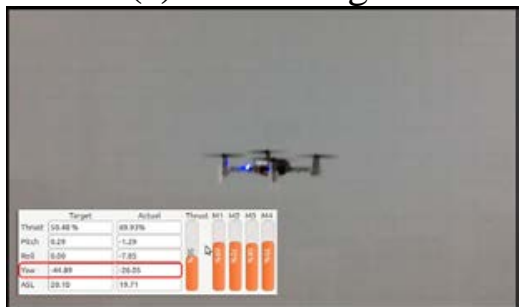

(f) yaw +45 degree/sec

\section{Conclusion}

In this research,we use the Leap Motion controller to read the data from the hand gestures, and transforms the gestures data into the corresponding control commands, sending them by Crazyradio PA module in order to control the flight attitude of Crazyflie 2 micro rotor craft. The coordinate transformation matrixes ensure the data consistency in different coordinate system transformation. In this paper, the control methods and the experimental platform of the aircraft control system are proposed, which can realize the interaction between gestures and aircraft flight attitude. Compared with the traditional way of the remote control aircraft, this kind of interaction is more direct in interaction, which is a extension of the man-machine interaction.

\section{Reference:}

1. Ren G, O’Neill E. : submitted to Journal of Computers \& Graphics (2013)

2. Information on http://www.leapmotion.com.

3. Coelho, Joanna C., and Fons J. Verbeek : Creating the Difference, Proceedings of the ChiSparks 2014 Conference, The Hague, The Netherlands.(2014)

4. Hantrakul L, Kaczmarek K. : Proceedings of the 2014 International Workshop on Movement and Computing. ACM, (2014): 142.

5. Bassily D, Georgoulas C, Guettler J, et al. : ISR/Robotik 2014; 41st International Symposium on Robotics; Proceedings of. VDE, (2014): 1-7.

6. Boyali A, Hashimoto N, Matsumato O. : SMART 2014, The Third International Conference on Smart Systems, Devices and Technologies. (2014): 20-25.

7. Kendoul F : submitted to Journal of Field Robotics (2012)

8. Weichert F, Bachmann D, Rudak B, et al. : submitted to Journal of Sensors (2013) 